\title{
Using Ecological Modeling to Enhance Instruction in Population Dynamics and to Stimulate Scientific Thinking
}

\author{
Hiraldo Serra ${ }^{1}$, Wesley Augusto Conde Godoy ${ }^{2}$ \\ ${ }^{1}$ College of Education, Federal University of Grande Dourados, Dourados, Brazil; \\ ${ }^{2}$ Department of Entomology and Acarology, "Luiz de Queiroz" College of Agriculture, University of São \\ Paulo, Piracicaba, Brazil. \\ Email: hiraldoserra@ufgd.edu.br, wacgodoy@esalq.usp.br \\ Received December 23 ${ }^{\text {rd }}, 2010$; revised February 15 $5^{\text {th }}, 2011$; accepted May $10^{\text {th }}, 2011$.
}

\begin{abstract}
Population dynamics has commonly been explored in high-school and undergraduate-level courses in ecology. The techniques used for teaching population dynamics can provide students with the required basic information for learning fundamental concepts in population ecology. However, population dynamics is a complex branch of population ecology that has an essentially quantitative nature. The effective assimilation of this topic should consider basic aspects of population theory, which involves the conceptual understanding of mathematical models. In this study, we propose an alternative methodology for teaching basic concepts of population ecology at the high-school and undergraduate levels, using mathematical models and numerical simulations on a microcomputer. We also show how an instructor or researcher can combine experimentation and theoretical ecology to produce simulations based on the ecology and biology of organisms. The study also suggests a way for teachers and professors to analyze population patterns with real data.
\end{abstract}

Keywords: Population Dynamics, Education, Alternative Methodology, Experimental Design, Mathematical Models

\section{Introduction}

Models play an essential role in all of the sciences. They may range from simple regression expressions to complex numerical simulations. Ecological systems are considered highly complex because they are characterized by diverse components, including nonlinear interactions, scale multiplicity, and spatial heterogeneity (Wu \& David, 2002). Ecological modeling explores how simulations can propose solutions to complex natural systems, such as biological populations (Lima, Ferreira, \& Godoy, 2009). With the advances in computation sciences, population modeling has become a subject of interest, and researchers have been considering didactic uses of simulations in recent years. Thompson, Simonson \& Hargrave (1996) defined simulation as a representation or model of some event, object, or phenomenon. Simulations may create several opportunities for learning, in different ways (Bell, Smetana, \& Binns, 2005). Students can obtain immediate feedback about complex phenomena and processes, and teachers are able to focus the students' attention on learning when systems are simplified by using mathematical models and simulations (Roughgarden, 1998). In the educational context, simulations can be a powerful technique to teach about important aspects of the world by modeling or replicating it (Alessi \& Trollip, 1991). However, students should not be motivated only by simulations or ecological models, but mainly by the questions that they involve and also by interacting with the models similarly to real situations. When systems become simpler, problems are more easily solved and processes are more understandable and easier to control (Javidi, 2004). Technological resources have grown rapidly in recent years, and nowadays teachers have easy access to computers and the Internet. Positive results have been reported from the use of modeling and simulations in the classroom, mainly with respect to the development of skills to solve problems and/or assess conceptual changes. Simulations arise from models, and therefore their reliability essentially depends on the model's foundations (Wu \& David, 2002). If the foundations are sufficiently solid, the reliability of the simulations is improved. It is important that models are built on real processes, mechanisms, and data (Hilborn \& Mangel, 1997). Data may give support to model simulations. In this context, it would be interesting to discuss how important data are for ecological modeling.

In this study, we offer an alternative methodology for use at the high-school level and also for undergraduate university students, to teach the basic concepts of population ecology using mathematical models and numerical simulations on a microcomputer, combined with laboratory experiments. The study also aims to provide teachers and professors with an alternative to show students the main phases of a research program involving data collection, analysis, and interpretation in a context of theoretical ecology, focusing on the behavior of theoretical patterns at the population level. The models presented are structurally simple, allowing rapid understanding of the system. Complexity is gradually introduced, making it possible to improve the fit between theory and reality. Essentially, three models are used to show basic ecological concepts: a density-independent population-growth model; a density-dependent model (logistic model); and a density-dependent model incorporating two density-dependent processes, fecundity $(F)$ and survival $(S)$, functions of immatures, $n_{t}$. This last model was developed to describe the population dynamics of flies, and in the current proposal their simulation results were obtained from real data (Godoy et al., 1993). Then, all the steps of the experimentation and modelling are shown, allowing an overall 
understanding of how to study a complex ecological process at the population level by using a simple tool. With these three models it is possible for readers to understand the main differences between ecological processes and define different theoretical structures for models, with emphasis on density dependence and population dynamics.

\section{Computer Simulations in Science Education}

Although conventional textbooks offer essential and interesting representations, simulations can provide opportunities to think about questions or phenomena in a more realistic way. For instance, after comparing simulated and hands-on dissection laboratory exercises, Akpan and Andre (1999) concluded, "The flexibility of these kinds of environments makes learning right and wrong answers less important than learning to solve problems and make decisions. Simulations promote learning about what-ifs and possibilities, not about certainties" (p. 118). The impact of simulations on the development of skills has been noted for different aspects, indicating that the design of experiments, measurements, and interpretation of data are more positively influenced by them than by traditional methods (Javidi, 2004).

\section{Mathematical Modeling in Education Research}

Areas that use mathematical models most intensively include physics, biology, electrical engineering, computer science, and the social sciences including economics, sociology and political science. Eykhoff (1974) defined a mathematical model as a representation of the essential aspects of an existing system (or a system to be constructed) which presents knowledge of that system in usable form. It is a technique for understanding the dynamics and/or patterns of a system, and also to make predictions about the system. Systems usually have two essential components: 1) elements that have certain qualities and properties, and 2) relationships and actions that explain how these elements interact and change (Norris, 1994). Indeed, mathematical models are descriptions of the system that they represent. Model users are generally able to investigate and understand the relationships between components of the system without having to actually manipulate it. Abstraction leads to the simplification of the system, but also to the gradual introduction of levels of complexity that are necessary to fit the model to reality (Varaki, 2006).

Description of behaviors, trends, or predictions generally involves concepts and processes, which can be symbolically incorporated into theoretical formulations that represent systems (Varaki, 2006). The active process of devising a mathematical model is called mathematical modeling (Breithach \& Maltas, 2003). Mathematical modeling is a systematic process that draws on many skills and employs the higher cognitive activities of interpretation, analysis, and synthesis. The modeling process includes the observation of phenomena, followed by the design of problems, as well as parameterization, a procedure that involves a choice of the variables and parameters connected to the problem. The relationships among factors should also be considered before applying the mathematical analysis and obtaining results. Once the results are obtained, refinements are needed in order to consolidate the model (Va- raki, 2006)

\section{Biological Population Dynamics}

Population dynamics has commonly been explored in Brazilian high schools as a part of the subject of ecology (Amabis $\&$ Martho, 2005), and in this context has involved interesting techniques for learning, which have been in regular use for several years. These techniques have provided students with the basic information required for learning fundamental concepts in population ecology. However, population dynamics is a complex branch of population ecology that is essentially quantitative in nature (Case, 2000). The effective assimilation of this topic should consider basic aspects of population theory, which involves the conceptual understanding of mathematical models (Bernstein, 2003).

Understanding of the theoretical formalism applied to dynamic systems requires a deeper knowledge of the structure of population models and a closer interaction among the student, the theoretical foundation, and the computer (Bernstein, 2003). Population mathematical models systematically analyze biological mechanisms, making it possible for the student to gain a general understanding of different natural systems, together with their processes, at different levels of complexity (Green et al., 2005). The structure of ecological communities, with many species interacting among each other and with the environment, includes complex relationships that basically involve organisms with symbiotic, host-parasite, competitor and predator-prey relationships (Murray, 2002). However, the comprehension of community structure requires analysis of single ecological systems initially, with only one or two species but with a mathematical perspective (Hastings, 1997).

At the population level, an issue of basic interest is the behavior of populations over a certain period of time, or in other words, what effects can population growth exerts on the dynamic behavior of populations? (Gotelli, 2001; Castanho et al., 2006). The identification of factors associated with the growth of biological populations constitutes a basic requirement to understand the regulatory mechanisms of population dynamics and communities (Royama, 1992; Schowalter, 2006). The quantitative study of the effects resulting from the spatio-temporal distribution of species in their habitats has recently become an important area of investigation for many ecologists (Cantrell \& Cosner, 2003).

\section{An Interdisciplinary Proposal for High-School and Undergraduate University Students}

We propose an alternative methodology for teaching population ecology and for promoting interaction between the student, theory, computer learning, and experimentation in population ecology. The theoretical foundation, computer simulations with mathematical models, and steps for setting up experiments connected to models are described below.

\section{Density-Independent Population Models}

A density-independent discrete population is analogous to a financial application, in that, $P_{0}$ is the initial deposit, $\mu$ is the income rate for the applied value, and $t$ is the interval in which 
the income is added to the applied initial value. Both situations, a biological population and a bank account, grow in a series of discrete steps, increasing more quickly while the number of organisms or the income can increase, because all individuals can contribute to generating new offspring.

Density-independent growth requires a simple model as a function of time, which describes changes in population size. Two variables, birth and immigration, influence the population input; and two, mortality and immigration, influence the outgo (Hastings, 1997). However, in order to simplify the system, migration will not be considered in this approach. A group of simple premises for a first stage could be:

1) Births and deaths lead to a balance for each population, determining its density;

2) All individuals are identical (especially in terms of the probability of death and of producing offspring);

3) The population consists of parthenogenetic organisms, simplifying the reproductive process;

4) Resources are infinite.

These assumptions simplify the population model and are instructive for the first stage of reflection. We compare two types of models to describe the population dynamics: geometric growth in discrete time, and exponential growth in continuous time.

\section{Geometric growth in discrete time}

The first density-independent growth model can be applied to many plants, insects, mammals, and other organisms with seasonal reproduction. Thus, these organisms can be pooled in the same cohort in the population. They receive newborns at an initial time, with the possibility of producing a new generation in a subsequent period. Because of mortality, the parents cannot live together with their offspring (e.g., annual plants) or with partial overlapping; they can survive to reproduce again (as with many mammals). The discrete population in time could be described by a finite difference equation, where:

$$
\begin{aligned}
& P_{t}=\text { population size at time } t \\
& b=\text { births per female at time } t \\
& s=\text { survival probability at time } t,
\end{aligned}
$$

$$
P_{t+1}=s P_{t}+s b P_{t}=(s+s b) P_{t}
$$

Rewriting the equation for the periods of birth and death, a single rate as a parameter that governs the population size would be $\mu=s+s b$, which gives the number of survivors of its offspring. Then:

$$
P_{t}=\mu P_{t-1}=\mu\left(\mu P_{t-2}\right)=\mu^{t} P_{0}
$$

In this equation, $\mu$ is the geometric growth rate and describes the temporal changes in terms of the number of individuals. For $\mu=1$, dead individuals are only replaced, indicating that the population size remains constant. For $\mu<1$, the population tends toward extinction, and for $\mu>1$, the population tends toward growth. With $\mu$ constant, the population size of future generations can be projected if the growth rate $(\mu)$, the initial population $\left(P_{0}\right)$, and the interval $(t)$ over which the growth occurs are known. Then,

$$
P_{t}=\mu^{t} P
$$

\section{Exponential growth in continuous generations}

Now we will consider organisms that reproduce continuously, similarly to humans or bacteria. Continuous population growth over time is best described by a differential equation, with the instantaneous rate defined infinitely over small intervals of time, where:

$P=$ population

$b=$ instantaneous birth rate per female

$d=$ instantaneous death rate per female

Then, the variation in population size is described as:

$$
\frac{\mathrm{d} P_{t}}{\mathrm{~d} t}=(b-d) P
$$

A single parameter can be used to express the birth and death rates $r=b-d$, and $r$ is called the intrinsic rate of increase or exponential growth rate. Then:

$$
\frac{\mathrm{d} P}{\mathrm{~d} t}=r N
$$

Population growth is proportional to $P$, and $r$ is the proportionality constant. When $r=0$, birth and death are in equilibrium, individuals only replace themselves, and the population size remains constant. When $r<0$, the population tends to extinction, and when $r>0$, the population grows. The quotient of variation for the population size is:

$$
\frac{\mathrm{d} P_{t}}{p \mathrm{~d} t}=r N
$$

The parameter $r$ is also called the per capita growth rate. Density-independent growth implies that the population growth rate per individual is constant. The differential equation for the continuous growth model (Equation (5)) can be integrated, to project future populations (analogously to Equation (3) for the discrete case).

$$
P_{t}=P_{0} \mathrm{e}^{r t}
$$

Although $r$ is the instantaneous rate, its numerical value is defined over a finite interval. If $r$ remains constant, it is possible to predict the future population size $\left(P_{t}\right)$, since we know the growth rate $(r)$, the initial population $\left(P_{0}\right)$, and the time over which the growth occurs $(t)$.

\section{Comparing Continuous and Discrete Density-Independent Growth Models}

If $\mu$ and $r$ are both constant growth rates, how can we relate them? To illustrate, we can calculate populations over different time intervals with both models. In the following comparison we assume that the interval for which a numerical value of $r$ for $\mu$ is defined, can be expressed by one generation.

Discrete Geometric Growth

$$
\begin{gathered}
P_{t}=\mu^{t} P_{0}=2 P_{0} \\
\mu^{t}=2 \\
t \ln (\mu)=\ln (2) \\
t=\frac{\ln (2)}{\ln (\mu)}
\end{gathered}
$$

Continuous Exponential Growth

$$
\begin{aligned}
r t & =\ln (2) \\
P_{t} & =\mathrm{e}^{r t} P=2 P_{0}
\end{aligned}
$$




$$
\begin{array}{r}
\mathrm{e}^{r t}=(2) \\
t=\frac{\ln (2)}{r} \\
\frac{\ln (2)}{\ln (\mu)}=\frac{\ln (2)}{r} \\
\mu=\mathrm{e}^{r}
\end{array}
$$

Applying the expressions 11 and 15 to two generations with the same interval, and solving $\mu$ in terms of $r$, or $r$ in terms of $\mu$, we obtain:

$$
\ln (\mu)=r
$$

Here, we show two ways to analyze density-independent population growth, in spite of the similarity between the models. The differences reflect a natural variation caused by real biological factors. In models that incorporate density-dependent feedback, the differences in life cycles affect the behavior of population dynamics.

Certainly, the geometric or exponential growth models do not describe population growth in the real world. With $\mu>1$ or $r>0$, the equations would give only mathematical descriptions of an outbreak (Gotelli, 2001). These models can provide only a partial description of population growth, since they are based on two assumptions that are rarely true: 1) all individuals are identical; 2) environmental resources will not become scarce if $r$ is constant.

Why do we present models that give descriptions of a system that are not very realistic? Because they illustrate the logical consequences of simple ideas. We can use simple models as a starting point, and then add terms to explore the complexities that bring them closer to the real situation. However, these relatively simple models are adequate to describe biological invasion processes that are successful in the initial periods (Hengeveld, 1989).

\section{Density-Dependent Population Growth (Logistic Model): A First Step for the Improvement of the Initial Models}

These models simulate density-dependent population growth, assuming a negative feedback for the population size, with the per capita growth rate described by a linear function. They require specification of the initial population size $\left(P_{0}\right)$, the sustainable maximum population size or environmental carrying capacity $(K)$, an intrinsic growth rate $(r)$, and a feedback interval $(I)$, optionally. The density-dependent models assume that population size influences the per capita growth rate. While the effect (feedback) of density on population growth can assume many forms, the logistic model imposes a negative linear feedback on the per capita growth rate.

If $K$ is the carrying capacity, then $(K-P)$ provides a measurement of the new carrying capacity, and $(K-P) / K$ describes the unused fraction of the carrying capacity, and then:

$$
\frac{\mathrm{d} P}{P \mathrm{~d} t}=r[K(K-P) / K]
$$

If $P$ is close to zero, not all resources are used, and $\mathrm{d} P / P \mathrm{~d} t$ is close to " $r$ ". If $P=K$, the resources are completely used, and $\mathrm{d} P / P \mathrm{~d} t=0$. Only in this differential equation continuous model, " $r$ " is an instantaneous rate and the defined numerical value in a finite time period. The finite difference equation produces a discrete analogous simulation for the continuous logistic model, written as:

$$
P_{t+1}=P \mathrm{e}^{r(1-P / K)}
$$

In this model, " $r$ " is the finite rate of increase. The model simulates the growth in density-dependent discrete generations, with no instantaneous feedback, and shows behaviors that cannot be detected by the continuous model, such as stable equilibrium, limit cycles, and chaos (Edelstein-Keshet, 1988).

The two types of models described above are the focus of the methodology proposed here. In this phase, students will be studying population models at different levels of abstraction, and will be able to focus them on different aspects. Teachers should have available a more in-depth explanation of the background, making it possible for them to understand the structure of the model at the level of deducing mathematical equations. Students will gain a more general view of the establishment of the biological premises that support the model, with formulations of the basic equations.

\section{Modeling with Excel}

The models can be built in Excel spreadsheets. For example, we will begin by simulating the dynamics with the density-independent growth model (Equation (3)). This equation describes the growth of a hypothetical population. Population size at time $t$ is the result of $P_{0}$ which is governed by the population growth rate $(\mu)$. Then with $u<1$, the population will decrease and tend toward extinction; with $u=1$, the population will remain constant; and with $u>1$, the population will show unlimited growth. This can easily be shown by using Excel, as follows. Type " $=10 * 0.4$ " in cell A1 and press "enter". The value 10 is the initial population size, and 0.4 is the growth rate $(u)$. Copy the result in A1 and paste in A2, replacing 10 with A1. A time series will be obtained by doing the same thing for ten or twenty cells below, from this point simply copying and pasting cells, without replacing any value. The graph will show the density-independent population $(P)$ dynamics at discrete time intervals (Figure 1).

A reverse behavior can be observed by setting $\mu=1.5$, for example (Figure 2). The population $(P)$ will show unlimited growth. It is possible to simulate exponential growth by using Equation (7), and also to use Equation (20) to evaluate the dynamics of the logistic model. The graph plotted from Equation (7) will show exponential growth; and if Equation (20) is used, a sigmoid curve will be produced, describing a population $(P)$ with its growth limited by the carrying capacity (Figure 3 ).

In order to produce Figure 3, type $P t, r$ and $K$ in cells A1, A2 and $\mathrm{A} 3$ respectively. Type $10,0.2$ and 100 in cells $\mathrm{B} 1, \mathrm{~B} 2$ and $\mathrm{B} 3$ respectively. In cell $\mathrm{C} 1$, type $=\$ B \$ 1 * \exp (\$ B \$ 2 *(1-$ $(\$ B \$ 1 / \$ B \$ 3))$ ) and copy. Paste this in cell $C 2$, replacing $\$ B \$ 1$ by $\mathrm{C} 1$. Paste the contents of $\mathrm{C} 2$ subsequently in the following cells down to cell $\mathrm{C} 20$, and plot the graph. The result will be a sigmoid curve, characterized by slow population growth in the first generations, followed by exponential growth and then saturation for the last generations (Figure 3). By changing the $r$ value to $1,2.2$ and 3 , it is possible to observe the different behaviors of the equation. By setting $r$ at 1 , the result will be an 


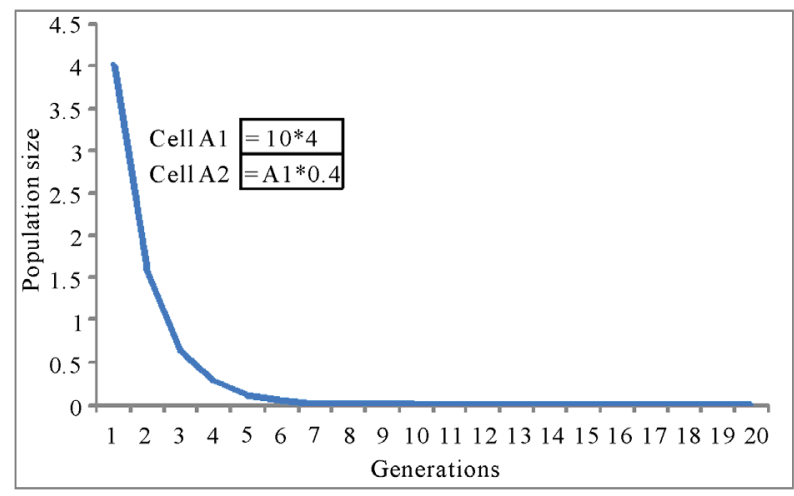

Figure 1.

Density-independent population $(P)$ trajectory with the discrete time model, showing a population decrease.

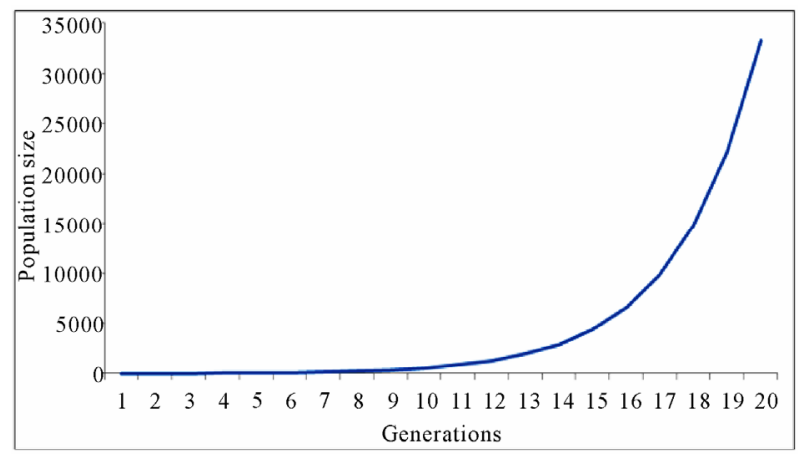

Figure 2.

Density-independent population (P) trajectory (Equation (7)) with the continuous time model, showing a population increase.

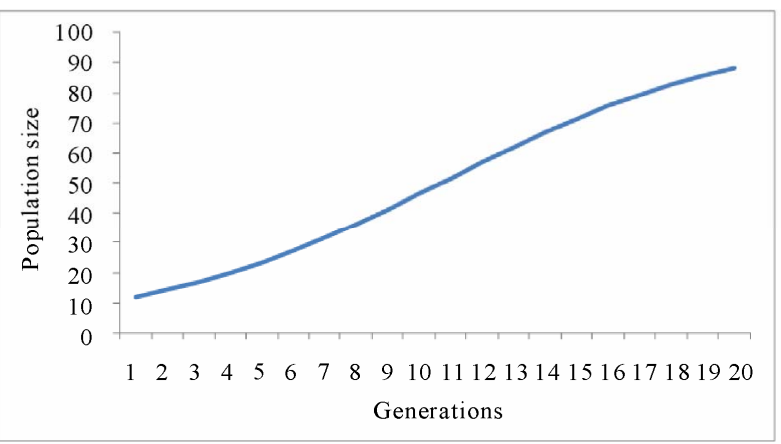

Figure 3.

Density-dependent population $(P)$ trajectory (Equation (20)) with logistic model showing population growth limited by the carrying capacity, with $r=0.2$.

anticipation of the curve saturation, characterized by the population reaching $K$, the carrying capacity (Figure 4).

If $r$ is set at 2, the population will show cycles (Figure 5). If $r$ is set at 3 , the cycles will be replaced by an unpredictable oscillation (Figure 6), which is usually termed "chaos" (Edelstein-Keshet, 1988). This variety of dynamic behavior in the simulations is a property of the model, but may also reflect important ecological patterns for populations, which can be discussed with the students. Fluctuating populations are proba- bly more susceptible to local extinction than stable populations, because they can reach zero suddenly ("crash") (Bernstein, 2003). This is an interesting point to discuss in the classroom. Changes in parameters are usually attributed to causes such as environmental conditions, especially temperature, humidity or

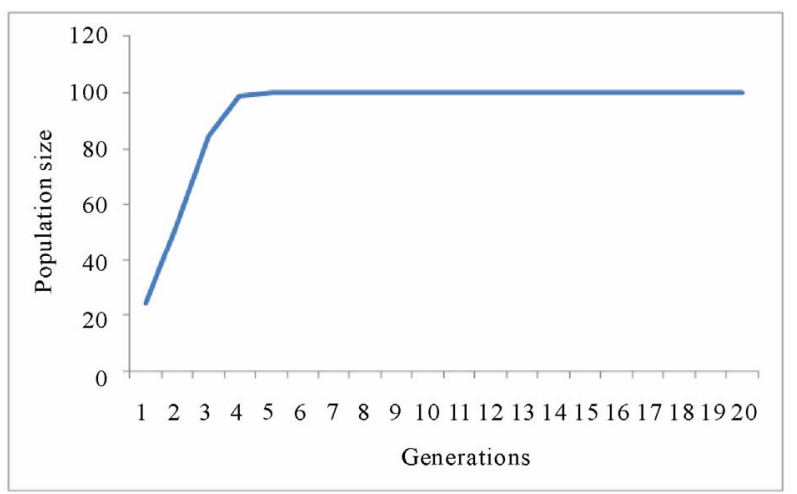

Figure 4.

Density-dependent population $(P)$ trajectory (Equation (20)) with logistic model showing population growth limited by the carrying capacity, with $r=1$.

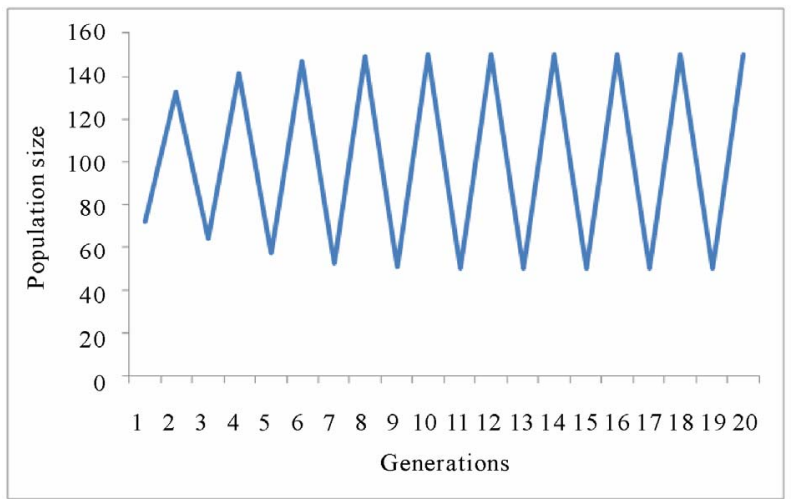

Figure 5.

Density-dependent population (P) trajectory (Equation (20)) with logistic model showing population growth limited by the carrying capacity, with $r=2.2$.

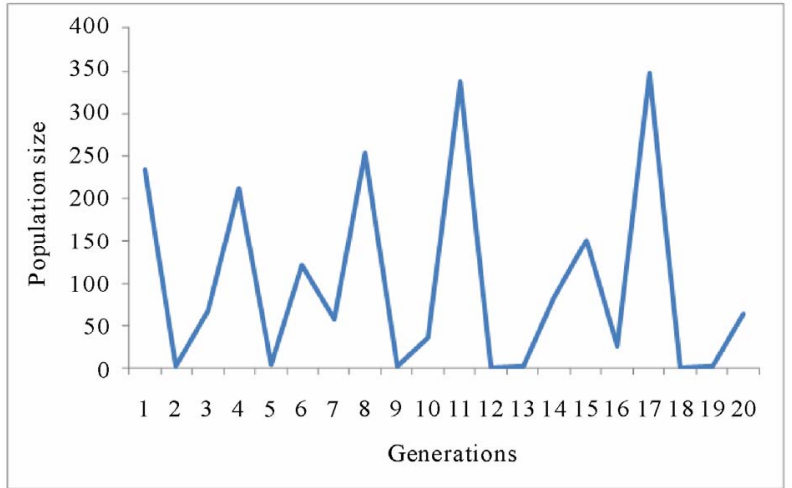

Figure 6.

Density-dependent population (P) trajectory (Equation (20)) with logistic model showing population growth limited by the carrying capacity, with $r=3.5$. 
rainfall. However, populations are also influenced by density-dependent processes, as noted previously for the other models (Figures 3 to 6 ). Figures 1 to 6 provide a basic understanding of how simple models can describe complex biological systems, with comparative descriptions of populations that are not limited and that are limited by the abundance of resources. Figures 3 and 4 show the trajectories of populations that reach carrying capacity.

Figures 4 to 6 show cycles that develop in response to resource scarcity, since they extrapolate the $K$ limit. In discrete time models, it is possible to find this variety of behaviors, differently from the continuous model, which shows frequently no time lag in the density-dependent response (Gotelli, 2001).

\section{Modeling a Real Biological System by Combining Experimentation with a Mathematical Model}

\section{Experimentation}

In this section, we demonstrate how to combine a real experiment with a mathematical model, by obtaining population data and using them to model the population growth of organisms. The organism used as an experimental model is a species of blowfly, Chrysomya putoria (Diptera: Calliphoridae). These flies have medical and veterinary importance, and are commonly found visiting decomposing organic substrates (Baumgartner \& Greenberg, 1984).

Blowflies can be easily collected by using baits such as rodent carcasses, dead fish, chicken viscera, or any other organic substrate of animal origin. By using a net it is possible to catch the adults flying over the substrate. After the adults are collected, they must be kept in cages $(30 \times 30 \times 30 \mathrm{~cm})$ covered with nylon mesh, and given water and sugar. An extra protein source such as fresh liver is necessary to allow the females to develop their ovaries. To obtain the eggs, ground beef can be used as a substrate. More details about blowfly collecting and rearing can be found in Godoy et al. (1993). Experiments are usually set up to analyze the population dynamics of flies, based on ecological processes that normally are observed on organic substrates. These provide perfect conditions for the blowflies to experience intraspecific competition for food.

Experiments can be performing by setting up larval densities in a fixed amount of artificial media (Godoy et al., 1993), ranging from 200 to 2000 larvae per vial, with increments of 200. Then, the population growth of $C$. putoria can be studied by investigating the sensitivity of demographic parameters such as fecundity and survival to increasing larval densities, such as 200, 400, 600, 800, 1000, 1200, 1400, 1600, 1800 and 2000. This range of densities is suitable to simulate intraspecific competition in flies, producing decreases in fecundity and survival as a function of density (Godoy et al., 1993). Experiments can show how fecundity and survival can depend on the density, characterizing an intraspecific competition for food, a common and important ecological process that can influence the population dynamics of blowflies (Godoy, 2007).

Fecundity is estimated by counting the number of eggs per female, expressed as the mean daily egg output, based on the length of the gonotrophic cycle of blowflies (Linhares, 1988). Survival is estimated as the number of adults emerging from each vial. The results of this experiment are analyzed by exponential regression, in order to generate the parameter values (Table 1) to use in a population growth model that was devel- oped to describe the population dynamics of flies (Godoy, 2007). The results from this experiment (Table 2) suggest that C. putoria shows a significant decrease in fecundity and survival as a function of larval density. This type of result is common in insects, which develop in discrete generations. Under high competition levels during the larval stage, many times these insects are not able to ingest enough food to develop satisfactorily in response to intraspecific competition. The result is that the insects show a decline in their parameter values. To learn the consequences of this decrease in values of demographic parameters for the population dynamics of $C$. putoria, it would be useful to find a mathematical model capable of describing the dynamics of the species in discrete generations. The density-dependent mathematical model proposed by Prout $\&$ McChesney (1985) is presented in the next section.

\section{Mathematical model}

The mathematical model developed by Prout and McChesney (1985) has been applied to analyze the population dynamics of blowflies (Godoy, 2007). The model simulates the population dynamics of flies, considering the number of immatures, in succeeding generations, $n_{t+1}$ and $n_{t}$. The model incorporates two density-dependent processes, fecundity $(F)$ and survival $(S)$, which are density-dependent functions of immatures, $n_{t}$. The recursion is written as a non-linear finite difference equation

$$
n_{t+1}=\frac{1}{2} F^{*} S^{*} \mathrm{e}^{-(f+s) n_{t}} n_{t}
$$

Table 1.

Parameters obtained from an exponential regression and used in the mathematical model.

\begin{tabular}{ccc}
\hline Intercept in $y$ & 0.97 & 19.32 \\
Regression coefficient & 0.00135 & 0.000569 \\
$t$ value & 8.3 & 26.44 \\
$r^{2}$ & 0.83 & 0.6 \\
ANOVA & 69 & 699 \\
\hline
\end{tabular}

Table 2 .

Mean daily fecundity and survival in response to larval density of $C$. putoria.

\begin{tabular}{ccccc}
\hline Density & $\mathbf{N}$ & Survival (\%) & $\mathbf{N}$ & Fecundity \\
\hline 200 & 2 & 66.75 & 60 & 17.7 \\
400 & 2 & 44 & 59 & 15.49 \\
600 & 2 & 49.91 & 59 & 15.92 \\
800 & 2 & 51.37 & 59 & 10.97 \\
1000 & 2 & 25.1 & 57 & 13.07 \\
1200 & 2 & 19.41 & 60 & 8.64 \\
1400 & 1 & 9.35 & 30 & 7.39 \\
1600 & 1 & 7.18 & 30 & 7.53 \\
1800 & 1 & 18 & 29 & 8,82 \\
2000 & 1 & 4.65 & 30 & 6.79 \\
\hline
\end{tabular}


where $F^{*}$ and $S^{*}$, the maximum theoretical fecundity and survival, are the intercepts in the exponential regression analysis. The factor $1 / 2$ indicates that only half of the population consists of adult females that contribute eggs to the next generation. The constants $f$ and $s$ are regression coefficients that estimate the slope of fecundity and survival on the density of immatures.

Equation (21) is a non-linear finite-difference equation, and its dynamics can be deduced by the single eigenvalue calculated at the point of equilibrium. The theoretical number of immatures at equilibrium $(k)$ is given by $n_{t+1}=n_{t}=k$, and this condition occurs when

$$
\frac{1}{2} F(k) S(k)=1
$$

The general expression for the eigenvalue associated with Equation (21) is given by the derivative of $n_{t+1}$ with respect to $n_{t}$ evaluated at $k$, which yields

$$
\lambda=1+\left.\frac{1}{2} k S(k) \frac{\mathrm{d} F\left(n_{t}\right)}{\mathrm{d} n_{t}}\right|_{n_{t}=k}+\left.\frac{1}{2} k F(k) \frac{\mathrm{d} S\left(n_{t}\right)}{\mathrm{d} n_{t}}\right|_{t}=k .
$$

If $\lambda<1$, the equilibrium is linearly stable. The number of immatures at equilibrium $(k)$ obtained from Equation (23) is given by

$$
k=\frac{\left(\ln \frac{F S}{2}\right)}{f+s}
$$

Finally, the eigenvalue $(\lambda)$ describing the stability at steady state is obtained from Equation (3) as

$$
\lambda=1-\frac{1}{2} k F^{*} f \mathrm{e}^{-f k} S(k)-\frac{1}{2} k S^{*} s \mathrm{e}^{-s k} F(k)
$$

\section{Prout's equation in Excel}

The dynamics of Equation (21) can be simulated in Excel by using real data. To do this, open a spreadsheet and type $F, S, s, f$ and $N t$ in cells A1, A2, A3, A4 and A5, respectively. In cells B1, B2, B3, B4 and B5, type the values 19.32, 0.97, 0.00135, 0.000569 and 200 respectively. These values can be found in Table 1, and reflect the fecundity, survival, and the respective regression coefficients estimated from the experimentally obtained data (Table 2). The last value, 200, is the initial population size. In cell $\mathrm{C} 1$, type $=0.5 * \$ \mathrm{~B} \$ 1 * \$ \mathrm{~B} \$ 2 * \mathrm{EXP}(-(\$ \mathrm{~B} \$ 3+$ $\$ B \$ 4) * \$ B \$ 5) * \$ B \$ 5$. This last expression is Prout's equation.

The first value, 0.5 , determines the population sex ratio, with $50 \%$ of the individuals being females. The symbol " $\$$ " used around letters maintains the parameter values held in the cells, without recurrence among cells. Copy cell $\mathrm{C} 1$ and paste in $\mathrm{C} 2$, replacing $\$ \mathrm{~B} \$ 5$ by $\mathrm{C} 1$. By doing this, we are creating conditions for recurrence among cells, i.e., the population at time $t+$ 1 is connected to the population at time $t$.

A time series will be obtained by doing same thing for 30 cells below; from this point, simply copy and paste the cells, without replacing any value. By selecting this column, plot a graph to see the population trajectory. The result can be interpreted as a two-point limit cycle (Figure 7), characterized by periodic oscillations bounded by two fixed values. It is possible to see significant alterations of dynamic behavior by merely changing the $F$ value. Change it to 30 and observe the new graph, which will show a four-point limit cycle. By changing $F$ to 40 , it is possible to find unpredictable oscillations, which are commonly termed chaos, similar to Figure 6 . The same thing can be done by changing the $S$ values in order to find other

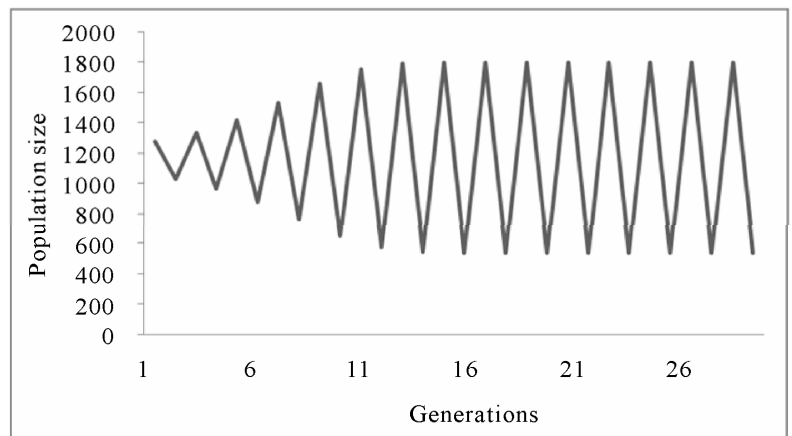

Figure 7.

Two-point limit cycle for C. putoria simulated with Prout's equation, with fecundity and survival as a function of larval density-dependence.

types of behaviors. This combination between experimentation and population theory shows students how sensitive the demographic parameters fecundity and survival are to changes in density. Interesting questions can be asked of the students, such as: which parameter, $F$ or $S$, is responsible for large changes in oscillation patterns? What can large changes in oscillation patterns produce in populations? Which is better for a population, a stable or an unstable dynamic? Why? The answers to these questions can be found by checking the literature cited in this paper. Some examples are the references (Roughgarden, 1998; Case, 2000; Hastings, 1997; Gotelli, 2001; Royama, 1992). This gives the student a new dimension to think about population growth, or about population dynamics in the context of conservation biology or pest control.

\section{Final Remarks}

Our proposal is supported by the literature that suggests that computers can play important roles in the classroom and laboratory science instruction, if they are well connected to a theoretical foundation and to real data (Bernstein, 2003). Computer simulations give students the opportunity to observe a realworld experience and to interact with it. Simulations are useful for simulating scenarios that are impractical, expensive, impossible, or too dangerous to run in real life (Baumgartner \& Greenberg, 1984). The literature suggests that the success of computer simulations used in science education depends on how they are incorporated into the curriculum and how the teacher uses them. Computer simulations are good tools to improve the students' skills in hypothesis construction, graphing, interpretation, and prediction.

\section{Acknowledgements}

HS hold fellowships awarded by CNPq. Thanks to anonymous referees for the useful suggestions. W.A.C.G. was partially supported by CNPq. The authors also thank Dr. Janet W. Reid for revising the English text.

\section{References}

Akpan, J. P., \& Andre, T. (1999). The effect of a prior dissection simulation on middle school students' dissection performance and understanding of the anatomy and morphology of the frog. Journal of Sci- 
ence Education and Technology, 8, 107-121. doi:10.1023/A:1018604932197

Alessi, S. M., \& Trollip, S. R. (1991). Computer based instruction: Methods and development. Upper Saddle River, NJ: Prentice Hall.

Amabis, J. M., \& Martho, G. R. (2005). Biologia de populações: Genética, evolução e ecologia. São Paulo: Moderna.

Baumgartner, D. L., \& Greenberg, B. (1984). The genus Chrysomya (diptera: Calliphoridae) in the new world. Journal of Medical Entomology, 21, 105-113.

Bell, R. L., Smetana, L., \& Binns, I. (2005) Simplifying inquiry instruction. The Science Teacher, 72, 30-33.

Bernstein, R. (2003). Population ecology: An introduction to computer simulations. Canada: John Wiley \& Sons Canada, Ltd.

Breithach, K., \& Maltas, J. (2003). An integrated curriculum in advanced mathematics/pre-calculus and physics. ULR (last checked 20 December, 2010) http://www.pls.uni. edu/couch/integrated philosophy.htm

Cantrell, R. S., \& Cosner, C. (2003). Spatial ecology via reactiondiffusion equations. Chichester: John Wiley and Sons.

Case, T. J. (2000). An illustrated guide of theoretical ecology. New York: Oxford University Press.

Castanho, M. J. P., Magnago, K. F., Bassanezi, R. C., \& Godoy, W. A. C. (2006). Fuzzy subset approach in coupled population dynamics of blowflies. Biological Research, 39, 341-352. doi:10.4067/S0716-97602006000200016

Edelstein-Keshet, L. (1988). Mathematical models in biology. New York, NY: Random House.

Eykhoff, P. (1974). System identification: Parameter and State Estimation. London: John Wiley \& Sons.

Godoy, W. A. C. (2007). Dynamics of blowfly populations. Functional Ecosystems and Communities, 1, 129-139.

Godoy, W. A. C., Reis, S. F., Von Zuben, C. J., \& Ribeiro, O. B. (1993). Population dynamics of Chrysomya putoria (wied.) (dipt. calliphoridae). Journal of Applied Entomology, 116, 163-169. doi:10.1111/j.1439-0418.1993.tb01184.x

Gotelli, N. J. (2001). A primer of ecology (3rd ed.). Sunderland, Massachusetts: Sinauer Associates, Inc.

Green, J. L., Hastings, A., Arzberger, P., Ayala, F. J., Cottingham, K. L., Cuddington, K., Davis, F., Dunne, J. A., Fortin, M. J., Gerber, L., \& Neubert, M. (2005). Complexity in ecology and conservation: Mathematical, statistical, and computational challenges. BioScience, $55,501-510$. doi:10.1641/0006-3568(2005)055[0501:CIEACM]2.0.CO;2

Hastings, A. (1997). Population biology. New York, NY: SpringerVerlag.
Hengeveld, R. (1989). Dynamics of biological invasions. New York, NY: Chapman \& Hall

Hilborn, R., \& Mangel, M. (1997). The ecological detective: Monographs in population biology. Princeton, NJ: Princeton University Press.

Javidi, G. (2004). A comparison of traditional physical laboratory and computer simulated laboratory experiences in relation to engineering undergraduate students conceptual understandings of a communication systems topic. Ph. D. Thesis, Florida: University of South Florida. (last checked 20 December, 2010) http://scholarcommons.usf.edu/etd/2936.

Lima, E. A. B. F., Ferreira, C. P., \& Godoy, W. A. C. (2009). Ecological modeling and pest population management: A possible and necessary connection in a changing world. Neotropical Entomology, 38, 699-707. doi:10.1590/S1519-566X2009000600001

Linhares, A. X. (1988). The gonotrophic cycle of chrysomya megacephala (diptera, calliphoridae) in the laboratory. Revista Brasileira de Entomologia, 32, 383-392.

Murray, J. D. (2002). Mathematical biology. Washington: Springer, Seattle.

Norris, D. (1994). Shortlist: A connectionist model of continuous speech recognition. Cognition, 52, 189-234. doi:10.1016/0010-0277(94)90043-4

Prout, T., \& McChesney, F. (1985). Competition among immatures affects their adult fertility: Population dynamics. American Naturalist, 126, 521-558. doi:10.1086/284436

Roughgarden, J. (1998). Primer of ecological theory. Upper Saddle River, New Jersey: Prentice Hall.

Royama, T. (1992). Analytical population dynamics. London: Chapman \& Hall.

Schowalter, T. (2006). Insect ecology. Orlando: Academic Press.

Thompson, A., Simonson M., \& Hardgrave, C. (1996). Educational technology: A review of the research (2nd ed.). Washington, DC: Association for Educational Communications and Technology.

Varaki, B. S. (2006). Math modeling in educational research: An approach to methodological fallacies. Australian Journal of Teacher Education, 31, 29-35.

Wu, J., \& David, J. L. (2002). A spatially explicit hierarchical approach to modeling complex ecological systems: Theory and applications. Ecological Modeling, 153, 7-26. doi:10.1016/S0304-3800(01)00499-9 